\title{
Rapid Determination of Full and Empty Adeno-Associated Virus Capsid Ratio by Capillary Isoelectric Focusing
} Tingting $\mathrm{Li}^{1,{ }^{*}}$, Tie $\mathrm{GaO}^{2}$, Hongxu Chen ${ }^{2}$, Peter Pekker ${ }^{3}$, Adrienn Menyhart ${ }^{3}$ and Andras
Guttman

\author{
${ }^{1}$ Sciex, Brea, CA, USA; ${ }^{2}$ AB Sciex, Beijing, China, ${ }^{3}$ Nanolab and ${ }^{4}$ Research Institute of Biomolecular and \\ Chemical Engineering, University of Pannonia, Hungary
}

\begin{abstract}
Adeno-associated virus (AAV) is one of the most promising gene transfer vector types featuring long-term gene expression and low toxicity. The lack of pathogenicity and the availability of many serotypes augmented the applicability of AAV virions in gene therapy applications. The recombinant AAV capsid includes the therapeutic protein-coding transgene as well as a promoter to initiate translation and a poly A sequence portion for stabilization. Current AAV manufacturing technologies, however, cannot guarantee the generation of only full capsids, i.e., including the entire required genome. Partially filled and empty capsids are also part of the product, decreasing in this way the efficacy and safety upon clinical translation. Therefore, rapid, accurate and QC friendly analysis of the full and empty capsid ratio is of high importance during AAV vector manufacturing and release testing. In this paper, an automated capillary isoelectric focusing technique is introduced, readily applicable in the biopharmaceutical industry for fast and efficient determination of the full and empty capsid ratio. The method also reveals information about the proportion of partially filled capsids. For higher resolution $(<0.1 \mathrm{pl}$ unit), mixtures of wide and narrow range ampholytes were utilized. The isoelectric point and peak area percentage reproducibility (RSD) of the mixed ampholyte assay were as low as $1.67 \%$ and $2.45 \%$, respectively, requiring only $65 \mathrm{~nL}$ of sample volume per injection.
\end{abstract}

ARTICLE HISTORY

Received: June 13, 2020 Revised: July 28, 2020

Accepted: August 02, 2020

DOI:

10.2174/1566524020666200915105456

CrossMark

Keywords: Adeno Associated Virus, capillary electrophoresis, Transmission electron microscopy, Isoelectric focusing, Full/empty capsid, Transgene.

\section{INTRODUCTION}

One of the most frequently utilized gene therapy vectors is Adeno-associated virus (AAV), featuring very low immunogenicity and different tropisms for multiple cell types $[1,2]$. The virus comprises a protein shell, also referred to as the capsid with an icosahedral shape of approximately 20-25 $\mathrm{nm}$ in diameter, composed of 60 viral protein subunits $[3,4]$. A singlestranded DNA molecule of approximately 4700-4800 bases is enclosed inside the capsid. This DNA in recombinant AAVs for gene therapy has three major elements, including the 145 nucleotide long inverted terminal repeats (ITR) at both ends, the promoter sequence and the therapeutic gene (transgene) [5]. During the manufacturing process of AAV vectors, many of the capsids either are not or are partially incorporated with the required DNA load (fragments or truncated). This can lead to a significantly low

*Address correspondence to this author at Sciex, Brea, CA, USA; E-mail: Tingting.Li@sciex.com production yield of $10-20 \%$, which results in a decrease in efficacy and an increase in potential safety risks. Hence, it is critical to be able to quantitatively determine the level of full, empty as well as partially filled AAV vectors.

Traditional methodologies used for the determination of the full vs empty AAV capsid ratios include spectrophotometry, analytical ultracentrifugation (AUC), electron microscopy (EM), ion-exchange chromatography (IEX), charge detection mass spectrometry (CDMS) as well as combined results obtained from both ELISA and qPCR data [6]. While some of these methods are well accepted and easy to use, they have limitations in accuracy and precision. Spectrophotometry based techniques use the UV absorbance ratio of the sample, measured at $260 \mathrm{~nm}$ and $280 \mathrm{~nm}$ to reveal the relationship between the protein to nucleic acid content [7]. While this approach is simple and fast, it suffers from impurity interferences and low accuracy. One of the broadly used analytical and preparative approaches capable of separating full, partially filled and empty capsids is analytical 
ultracentrifugation [8]. AUC has a large sample requirement and is difficult to utilize as a rapid quality control (QC) release assay system. Transmission and cryo-electron microscopy, as well as atomic force microscopy [9-12] are other frequently used methods capable of counting the number of full and empty capsids in a sample population, however, these methods have difficulties in determining the extent of DNA content in partially filled capsids. In addition, the evaluation of electron microscopy data is timeconsuming, making it less desirable in the $Q C$ and release testing environment. EM methods also heavily rely on the analyst's interpretation of the data, which can be somewhat subjective. lon-exchange chromatography (IEX) is a quantitative but lowresolution technique for full and empty capsid ratio determination $[13,14]$. IEX also requires larger sample amounts and suffers from serotype dependence. Mass spectrometry (MS) based approaches, such as charge detection MS, can readily detect full, partially filled and empty capsids, yet commercially not available for routine use [15].

The drawbacks of the above listed techniques called for more comprehensive, fast, easy to perform, robust approaches that are appropriate for QC/release testing to quantify the full and empty AAV capsid ratio with the possibility to reveal information on partially filled species, AAV charge heterogeneity and impurities. Capillary isoelectric focusing (cIEF) is a well-established bioanalytical technique in the biopharmaceutical industry, capable of separating closely related species, based on their isoelectric points, such as charge variants [16]. Due to their negatively charged DNA load, full AAV capsids generally have lower isoelectric points compared to their empty or partially filled counterparts [17], making CIEF a promising tool to fill the gaps described above. In this paper, we introduce an automated capillary isoelectric focusing method, featuring low sample volume requirements in conjunction with the use of narrow $\mathrm{pH}$ range ampholytes ( $\leq 0.1 \mathrm{pH}$ unit resolution) for rapid ( $<45 \mathrm{~min}$ ) QC friendly analysis of full, empty and partially filled AAV capsids.

\section{MATERIALS AND METHODS}

\subsection{Chemicals}

Phosphoric acid, sodium hydroxide, acetic acid, Larginine, iminodiacetic acid and all other chemicals were from Sigma-Aldrich (St Louis, MO). AAV samples were from SignaGen Laboratories (Rockville, MD) and Vigene Biosciences (Rockville, MD) with the serotypes specified below. The components of the Advanced ClEF Starter Kit were used with the $\mathrm{N}-\mathrm{CHO}$ coated capillary (Sciex, Framingham, MA), unless specified otherwise. All solutions were filtered through $5 \mu \mathrm{m}$ syringe filters prior to use.

\subsection{Sample Preparation}

The master-mix solution was prepared by combining $200 \mu \mathrm{L}$ of $3 \mathrm{M}$ urea - clEF gel solution, 12 $\mu \mathrm{L}$ of ampholytes ( $\mathrm{pH}$ range specified in the relevant sections), $20 \mu \mathrm{L}$ of cathodic stabilizer $(500 \mathrm{mM} \mathrm{L}-$ Arginine), $2 \mu \mathrm{L}$ of anodic stabilizer $(200 \mathrm{mM}$ iminodiacetic acid) and $2 \mu \mathrm{L}$ of each pl marker used. The following AAV serotypes were used in the experiments from SignaGen Laboratories (Rockville, MD): AAV1-CMV-GFP (titer: $1.46 \times 10^{13} \mathrm{GC} / \mathrm{mL}$ ), AAV3Cre-GFP (titer: $1.02 \times 10^{13} \mathrm{GC} / \mathrm{mL}$ ), AAV5-CMV-GFP (titer: $1.53 \times 10^{13} \mathrm{GC} / \mathrm{mL}$ ) and AAV9-CMV-GFP (titer: $3.12 \times 10^{13} \mathrm{GC} / \mathrm{mL}$ ). AAV8 of pAV-CMV-GFP Empty Capsid sample with a titer of $5.10 \times 10^{12} \mathrm{GC} / \mathrm{mL}$ was used as enriched empty capsids, while packaged AAV8 of the pAV-CMV-GFP sample with a titer of $1.10 \times 10^{13}$ $\mathrm{GC} / \mathrm{mL}$ as enriched full capsids, both from Vigene Biosciences (Rockville, MD). $3 \mu \mathrm{L}$ of AAV samples were mixed with $24 \mu \mathrm{L}$ of master mix and transferred into nanoVials (Sciex) for cIEF analysis. Either the Pharmalyte, 3-10 wide $\mathrm{pH}$ range ampholyte (GE Healthcare) alone, or its mixtures with SERVALYT 6-8 narrow pH ampholyte (Serva, Heidelberg, Germany) or $\mathrm{SH}$ AESlytes 7-8 narrow pH ampholyte (Advanced Electrophoresis Solutions, Cambridge, Ontario) were used at various ratios to fine-tune resolution.

\subsection{Capillary Isoelectric Focusing (cIEF)}

All ClEF experiments were performed in normal polarity separation mode (anode at the injection side) on a PA 800 Plus Pharmaceutical Analysis system (SCIEX), equipped with a UV detector (280 $\mathrm{nm}$ filter). The separation capillary was filled with the relevant ampholyte solutions, as defined in the 'Results and Discussion' section. The anolyte was $200 \mathrm{mM}$ phosphoric acid, while the catholyte was $300 \mathrm{mM}$ sodium hydroxide. The chemical mobilizer was $350 \mathrm{mM}$ acetic acid and the capillary cleaning solution was 4.3 $\mathrm{M}$ urea. The cathodic and anodic stabilizer solutions were $500 \mathrm{mM} \mathrm{L}$-Arginine and $200 \mathrm{mM}$ iminodiacetic acid (IDA), respectively. The $\mathrm{N}-\mathrm{CHO}$ coated capillary was used for all ClEF analyses and the separation temperature was maintained at $20^{\circ} \mathrm{C}$. The data were collected and analyzed by the 32 Karat Software version 10.3 .

\subsection{Transmission Electron Microscopy (TEM)}

One drop $(\sim 5 \mu \mathrm{l})$ of AAV5 sample was placed onto a TEM copper grid covered by a thin (3-5 nm) continuous carbon film. The excess sample was soaked up with a paper tissue. Phosphotungstic acid $(2 \%)$ was used for staining the sample for 30 seconds. A $200 \mathrm{kV}$ FEl Talos F200X transmission electron microscope (equipped with a field-emission gun) was used for the measurements (Thermo Fisher, Waltham, MA). The TEM bright-field images were taken at random places and constant magnification ( $1 \mu \mathrm{m}$ field of view) with a 4096 x 4096 pixel CMOS camera (FEI CETA). The evaluation was made by manual counting of at least 5000 virus particles.

\section{RESULTS AND DISCUSSION}

A pilot experiment was conducted first to understand the CIEF migration behavior of enriched full 
and enriched empty AAV capsids, shown in Fig. 1. In this experiment, the wide $\mathrm{pl}$ range ampholyte $(\mathrm{pH} 3-10)$ was used in the separation capillary with sodium hydroxide in the cathode and phosphoric acid in the anode reservoirs. The upper trace depicts the electropherogram of the enriched full capsid sample. As one can observe, besides the peak corresponding to the full capsid (peak 3 ), a few transitional products were also detected (peak group 2) along with a small peak representing, in this case, the empty capsid (peak 1), all focused between the $\mathrm{pH} 7.0$ and $10.0 \mathrm{pl}$ markers. The lower trace shows the separation of the sample containing the enriched empty capsid. Similar to the upper trace, peak 1 represents the empty, while peak group 2 represents the various partially filled transitional capsids and peak 3 the full capsid. These results suggested that the empty capsid focused at a higher pl value, while the full capsid at a lower pl value, the latter due to its negatively charged DNA load. The partially filled capsids focused between the empty and full capsid peaks, corresponding to the pl values defined by their actual DNA content.

After verifying the clEF focusing order of the empty and full adeno associated virus capsids, five commercially available AAV samples of different serotypes (AAV1, AAV3, AAV5, AAV8 and AAV9) were analyzed using the wide $\mathrm{pH}$ range ampholyte $(\mathrm{pH} 3-10)$ as shown in Fig. 2. Trace (a) depicts a blank run, while the upper traces of (b-f) show the corresponding AAV serotype associated electropherograms. The pl values of the separated peaks were determined by a calibration plot using a pl marker mixture and were found as follows: $\mathrm{AAV} 1=\mathrm{pl}$ 7.8-8.0; $\mathrm{AAV} 3=\mathrm{pl}$ 8.7-9.0; AAV5 = pl 7.0-7.2; AAV8 = pl 7.0-7.5; AAV9 = pl 7.37.5. Analysis of the AAV1 sample (trace b) revealed an overlapping peak distribution of the differently loaded capsids, i.e., empty, partially filled and full. The separation of the AAV3 (trace c) and AAV5 (trace d) samples featured two main peaks and a smaller one in between, considered to be corresponding to the empty, partially filled and full capsids. Again, the fact that the full capsids have lower pl values is due to the large amount of negative charges carried by the encapsulated DNA. AAV8 (trace e) and AAV9 (trace f) capsids focused on single broad peaks (the latter one with a shoulder), suggesting poor resolution of the variously filled capsid forms of these serotypes with the use of the wide $\mathrm{pH}$ range ampholyte.

As one can observe in Fig. 2, with the use of the wide $\mathrm{pH}$ range ampholyte, the analysis of the AAV5 sample showed the well-resolved separation of the empty, partially filled and full capsid peaks (trace d). The full and empty percentage distribution of this sample from the processed CIEF data was determined as $43 \%$ and $57 \%$, respectively. The calculation was made by applying the correction factor introduced by

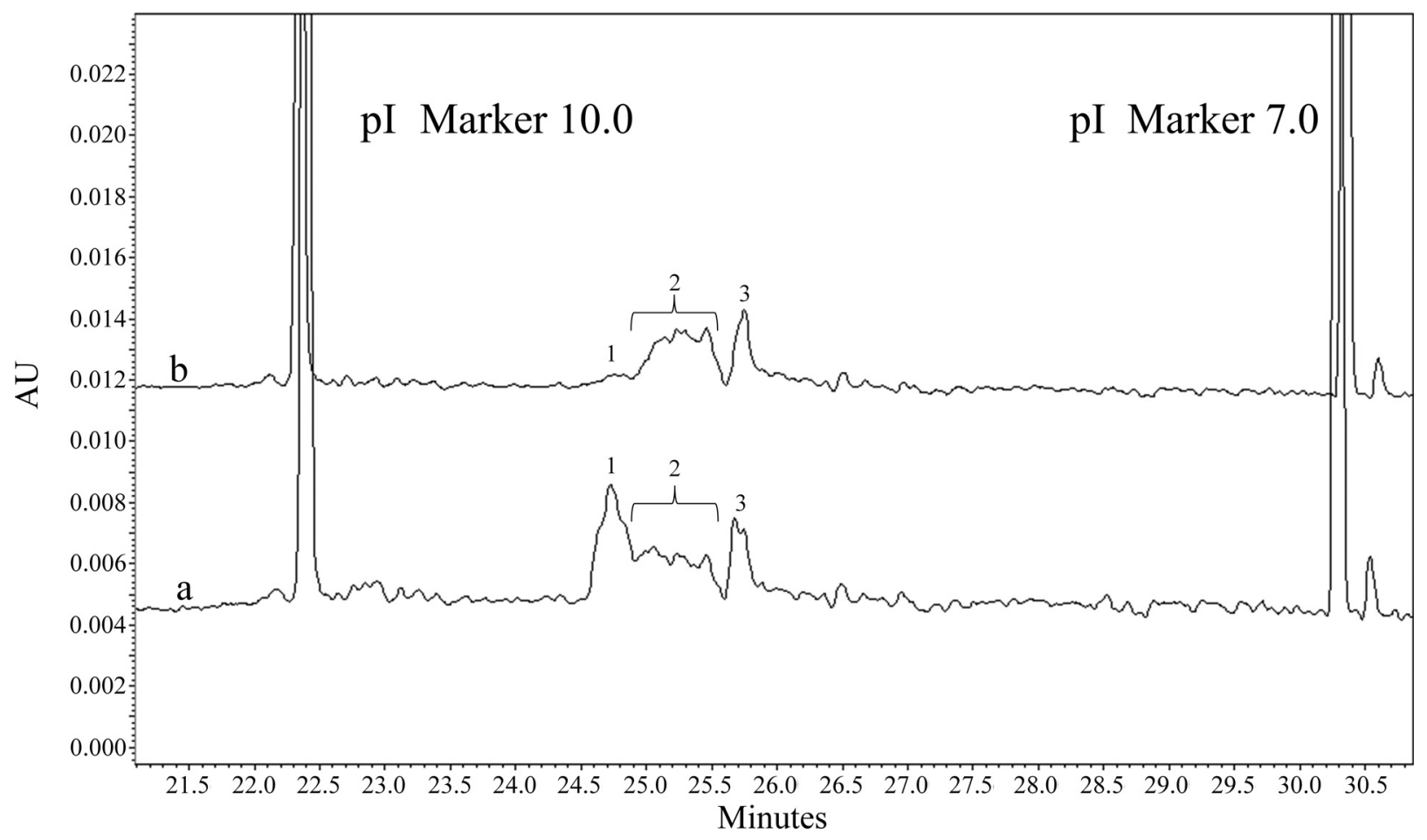

Fig. (1). Capillary isoelectric focusing of enriched empty (trace a) and enriched full (trace b) AAV capsids for migration/focusing order determination. Peaks: 1-empty, 2-transitional and 3-full capsids. Conditions: $20 \mathrm{~cm}$ effective length N-CHO capillary (50 $\mu \mathrm{m}$ ID and $30 \mathrm{~cm}$ total length), filled with 3-10 wide $\mathrm{pH}$ range ampholyte containing background electrolyte. Catholyte: $300 \mathrm{mM}$ sodium hydroxide, Anolyte: $200 \mathrm{mM}$ phosphoric acid. Applied electric potential: $25 \mathrm{kV}$ for the 15 min for the focusing step. Chemical mobilization step: $30 \mathrm{kV}$ with $350 \mathrm{mM}$ acetic acid for $30 \mathrm{~min}$ from the outlet reservoir. The separation temperature of the capillary cartridge was set at $20^{\circ} \mathrm{C}$. (A higher resolution / colour version of this figure is available in the electronic copy of the article). 


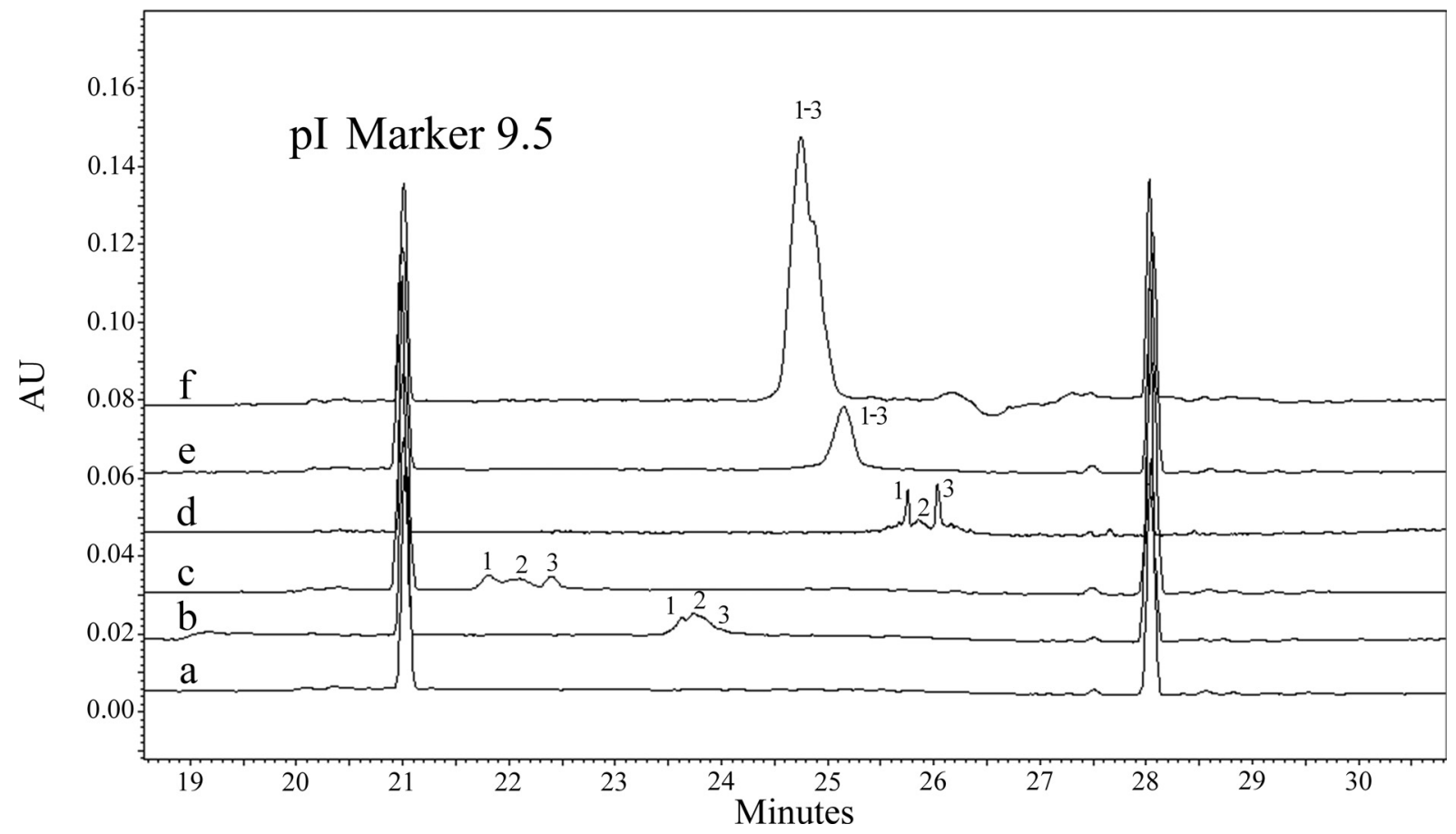

Fig. (2). Capillary isoelectric focusing analysis of five AAV capsid samples with different serotypes using the wide $\mathrm{pH}$ range ampholyte of pH 3-10. Traces: a) Blank, b) AAV1, c) AAV3, d) AAV5, e) AAV8 and f) AAV9. Peaks: 1-empty, 2-partially filled and 3-full capsids. Conditions were the same as in Fig. 1. (A higher resolution / colour version of this figure is available in the electronic copy of the article).

Goricar et al., [18] to alleviate any possible overestimation of the amounts of the full capsids that might have been caused by the $280 \mathrm{~nm}$ UV detection. This particular serotype was also subject to analysis by transmission electron microscopy (TEM), an orthogonal AAV capsid characterization method. Figure 3 shows a representative section of the TEM results, where the green circles and squares depict examples of full and empty capsid images, respectively. The arrows show the transitional capsids. The full to empty distribution using this method was assessed as $35 \%$ to $65 \%$, by manually counting the capsid types, i.e., reasonably similar to that obtained with the clEF method.

To quantify the capsid load distribution in some of the serotypes that resulted in either broad co-focused (AAV1) or non-separated single peaks (AAV8 and 9) with the use of the wide range ampholyte of $\mathrm{pH} 3-10$, the resolution of the analysis was fine-tuned by mixing the wide range type with narrow range ones. Trace (a) in Figure 4 depicts the results obtained with the use of $1: 1$ ratio of the wide range $(\mathrm{pH} 3-10)$ and narrow range (pH 6-8) ampholytes for the analysis of the AAV1 sample. As one can observe, in contrast to the results obtained with the use of the wide range ampholyte only (Fig. 2, trace b), this particular sample was resolved into five separated peaks, apparently corresponding to the empty (peak 1), partially filled (peak 2) and full (peak 3 ) capsids. Peaks 4 and 5 are probably sample related impurities. The middle trace (b) in Fig. 4 shows the separation of the AAV8 capsid sample with the 2:1 ratio of the wide and narrow range ampholytes resulting in a significant resolution increase in comparison to trace e in Fig. 2. The multiple peaks were obtained assumable corresponding to the empty

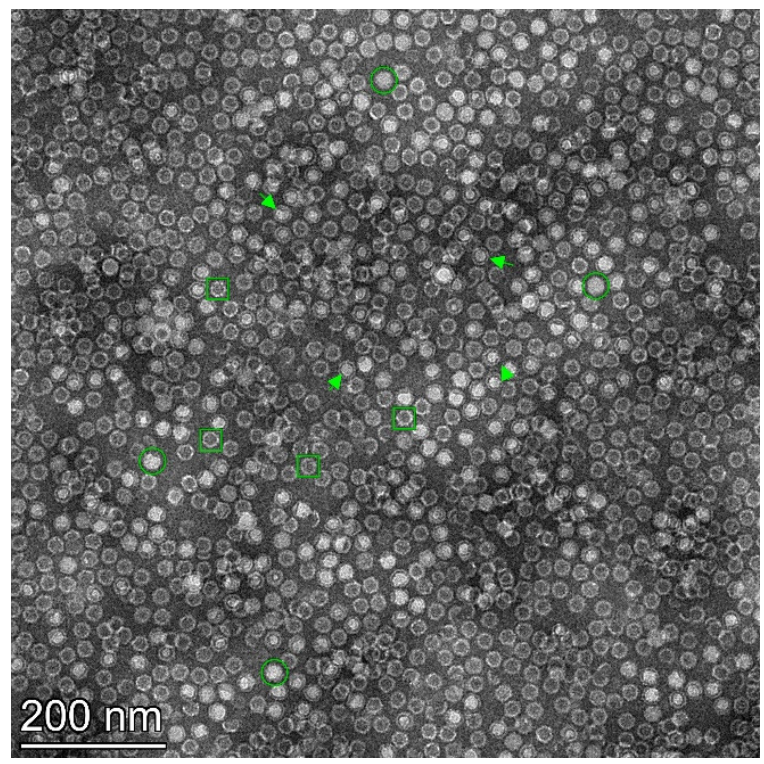

Fig. (3). Analysis of the full and empty capsid distribution of the AAV5 sample by transmission electron microscopy (TEM). Green Circles: examples of full capsid images; Green Squares: examples of empty capsid images; Green Arrows: examples of transitional capsids. Conditions: TEM: $200 \mathrm{kV}$ FEI Talos F200X transmission electron microscope with bright-field images taken at random places and constant magnification ( $1 \mu \mathrm{m}$ field of view) with a $4096 \times 4096$ pixel CMOS camera. ( $A$ higher resolution / colour version of this figure is available in the electronic copy of the article). 
(peak 1), partially filled (peaks 2 and 3 ) and full (peak 4) capsids. Another example of the usefulness of such $\mathrm{pH}$ range optimization is delineated in the upper trace of Fig. 4 (c), where a good separation of the AAV9 sample is shown with the use of 2:1 ratio mixture of the wide and narrow range ampholytes. Please note when using the wide range ampholyte alone for this particular sample, only a single peak with a shoulder was observed (Fig. 2, trace f). However, applying the 2:1 mixture of $\mathrm{pH} 3-10$ and 6-8 ampholytes resulted in excellent separation of all major components, probably corresponding to the empty (peak 1), partially filled (peaks 2 and 3 ) and full (peak 4) capsids. Peak 5 here was also considered as sample related impurity. This particular sample apparently contained mostly partially filled capsids, with various DNA loads represented by the individual peaks of 2 and 3 ). The assay reproducibility was also evaluated for this sample based on 6 consecutive runs and the pl and peak area percent RSD values were 1.67 and $2.45 \%$, respectively.

Interestingly, in some instances, further CIEF optimization did not bring about better separations. As an example, additional optimization by mixing the $\mathrm{pH} 3$ 10 ampholyte with an ultra-narrow range type of $\mathrm{pH} 7-8$ in 2:1 ratio (Fig. 5, trace b) did not result in better separation of the AAV5 serotype as with the use of the 2:1 ratio of $\mathrm{pH} 3-10$ and $\mathrm{pH}$ 6-8 ampholyte mixture (trace a). Albeit, trace (b) in this example shows 9 peaks, some of them might be just focusing on artifacts or spikes (e.g., peaks 2, 3 and 4). Trace (a), on the other hand, while featuring only five peaks, can be better evaluated from cIEF point of view with the empty (peak 1), full (peak 5) and partially filled (peaks 2-4) capsids.

\section{CONCLUSION}

In this paper, an automated cIEF based rapid and high-resolution method was introduced to determine the full/empty capsid ratios of different serotype AAV vectors. The full capsids focused at the lower, while the empty ones at the higher pl regime, respectively, based on their DNA load. In addition, the generated data also provided information about the presence of partially filled capsids, focused in between. Mixing wide and narrow range ampholytes resulted in better separation of otherwise co-focusing full, transitional and empty capsids with very small pl value differences. The reproducibility of the assay, even with the mixed range ampholytes was as low as $1.67 \%$ RSD for isoelectric point and $2.45 \%$ for peak area percentage determination. It is also important to note that the clEF method required less than $5 \mu \mathrm{L}$ of the total sample size for multiple runs, considering the typical injection volume of $65 \mathrm{~nL}$.

\section{LIST OF ABBREVIATIONS}

$\begin{array}{lll}\mathrm{AAV} & = & \text { Adeno Associated Virus } \\ \mathrm{AUC} & = & \text { Analytical ultracentrifugation } \\ \mathrm{CIEF} & = & \text { capillary isoelectric focusing } \\ \mathrm{GC} & = & \text { genome copy }\end{array}$

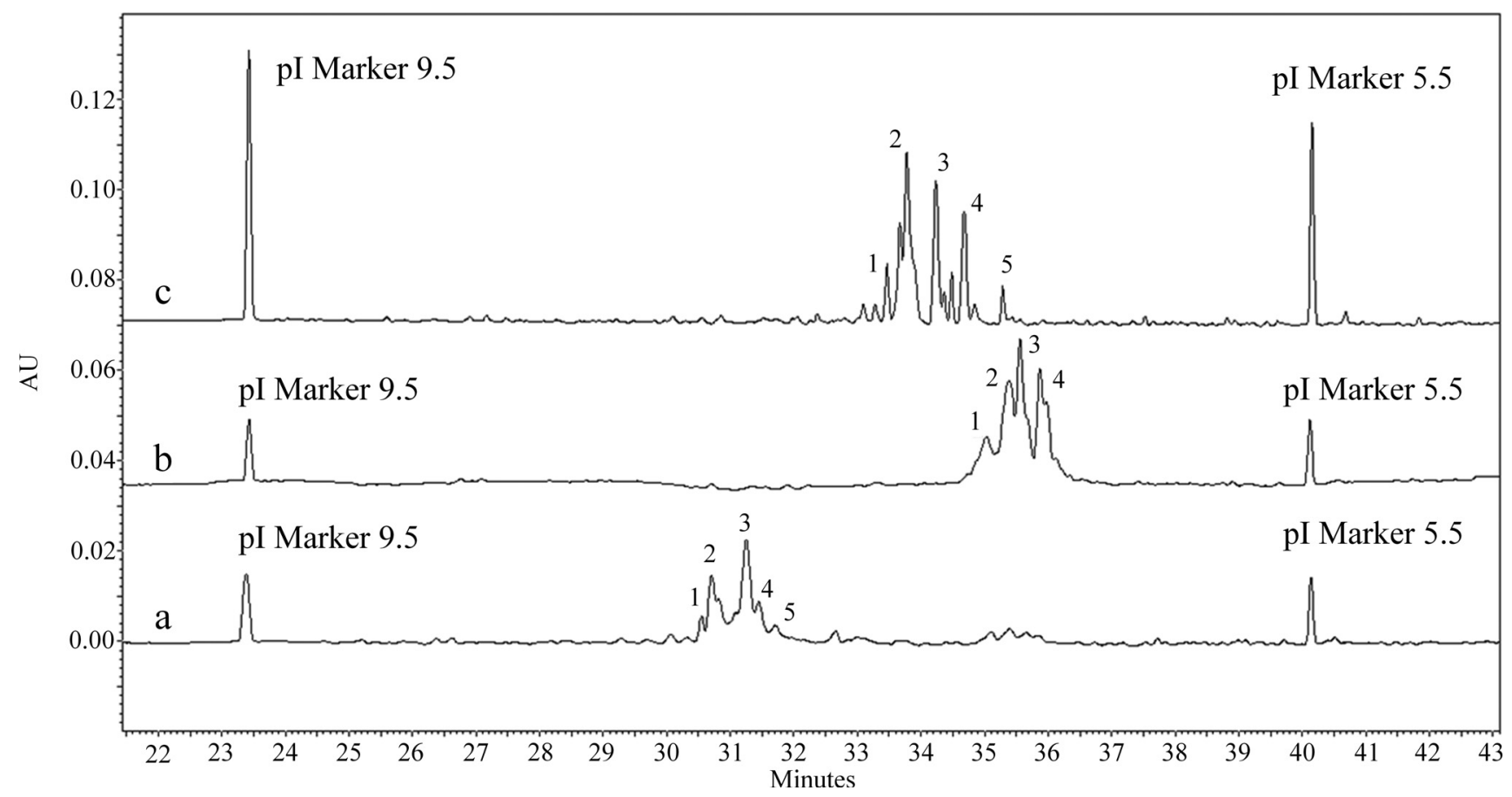

Fig. (4). Optimization of the different $\mathrm{pH}$ range ampholyte mixtures (pH 3-10 and $\mathrm{pH}$ 6-8) for high-resolution clEF analysis of the AAV1, 8 and 9 serotypes. Traces: a) AAV1 with 1:1, b) AAV8 with 2:1 and 3) AAV9 with 2:1 mixture of wide and narrow range ampholytes. Peaks: 1-empty, 2,3-partially filled, 4-full capsids, 5-sample related impurity. All other conditions were the same as in Figure 1. (A higher resolution / colour version of this figure is available in the electronic copy of the article). 


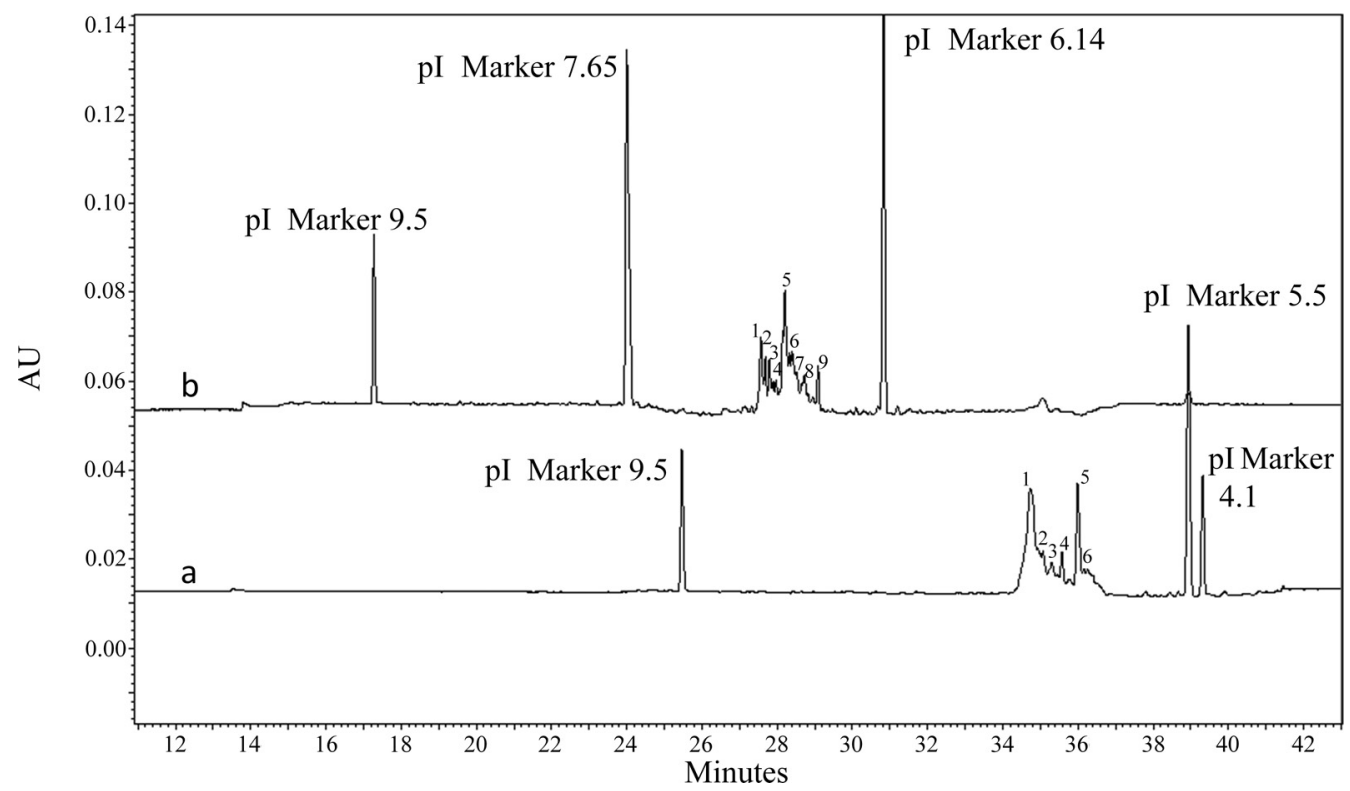

Fig. (5). Effect of the addition of an ultra-narrow range ampholyte on the resolution of the cIEF analysis of the AAV5 serotype using 2:1 ratios of the $\mathrm{pH}$ 3-10 plus $\mathrm{pH}$ 6-8 (trace a) and the $\mathrm{pH}$ 3-10 plus pH 7-8 (trace b) mixtures. Peaks in trace a) 1: empty, 2-4: partially filled, 5: full; trace b) 1: empty, 2-4: empty related focusing artefacts, 5-8: partially filled and 9: full capsids. Conditions were the same as in Figure 1, with the exception of the ampholyte mixtures used. (A higher resolution / colour version of this figure is available in the electronic copy of the article).

IEX = ion exchange chromatography

TEM = Transmission electron microscopy

\section{ETHICAL APPROVAL AND CONSENT TO PARTICIPATE}

Not applicable

\section{HUMAN AND ANIMAL RIGHTS PARTICIPATE}

No animals/humans were used for studies that are the basis of this research.

\section{CONSENT FOR PUBLICATION}

Not applicable.

\section{AVAILABILITY OF DATA AND MATERIALS}

Not applicable.

\section{FUNDING}

TEM was performed in the Nanolab, University of Pannonia, established using grant no. GINOP-2.3.3-152016-0009 from the European Structural and Investments Funds and the Hungarian Government

\section{CONFLICT OF INTEREST}

The authors declare no conflict of interest, financial or otherwise.

\section{ACKNOWLEDGEMENTS}

The useful discussions with Prof Mihaly Posfai and Dr Laszlo Hajba are greatly appreciated.

\section{REFERENCES}

[1] Lu Y. Recombinant adeno-associated virus as delivery vector for gene therapy--a review. Stem Cells Dev 2004; 13(1): 13345.

http://dx.doi.org/10.1089/154732804773099335 PMID 15068701

[2] Coura RdosS, Nardi NB. The state of the art of adenoassociated virus-based vectors in gene therapy. Virol J 2007; 4: 99.

http://dx.doi.org/10.1186/1743-422X-4-99 PMID: 17939872

[3] Naso MF, Tomkowicz B, Perry WL III, Strohl WR. AdenoAssociated Virus (AAV) as a Vector for Gene Therapy. BioDrugs 2017; 31(4): 317-34.

http://dx.doi.org/10.1007/s40259-017-0234-5 PMID: 28669112

[4] Cook KS, et al. Vaccine Plasmid Topology Monitoring by Capillary Gel Electrophoresis Curr Mol Med 2020; 20(10): 798-805.

[5] dos Santos Coura R, Beyer Nardi N. A role for adenoassociated viral vectors in gene therapy. Genet Mol Biol 2008; 31(1): 1-11. http://dx.doi.org/10.1590/S1415-47572008000100001

[6] Dobnik D, Kogovšek P, Jakomin $\mathrm{T}$, et al. Accurate Quantification and Characterization of Adeno-Associated Viral Vectors. Front Microbiol 2019; 10: 1570. http://dx.doi.org/10.3389/fmicb.2019.01570 PMID: 31379763

[7] Sommer JM, Smith PH, Parthasarathy S, et al. Quantification of adeno-associated virus particles and empty capsids by optical density measurement. Mol Ther 2003; 7(1): 122-8. http://dx.doi.org/10.1016/S1525-0016(02)00019-9 PMID 12573625

[8] Burnham B, Nass S, Kong E, et al. Analytical Ultracentrifugation as an Approach to Characterize Recombinant Adeno-Associated Viral Vectors. Hum Gene Ther Methods 2015; 26(6): 228-42.

http://dx.doi.org/10.1089/hgtb.2015.048 PMID: 26414997

[9] Chen H. Comparative observation of the recombinant adenoassociated virus 2 using transmission electron microscopy and atomic force microscopy. Microsc Microanal 2007; 13(5): 384-9. 
[10] Kronenberg S, Kleinschmidt JA, Böttcher B. Electron cryomicroscopy and image reconstruction of adeno-associated virus type 2 empty capsids. EMBO Rep 2001; 2(11): 9971002.

http://dx.doi.org/10.1093/embo-reports/kve234 PMID: 11713191

[11] Lerch TF, O'Donnell JK, Meyer NL, et al. Structure of AAVDJ, a retargeted gene therapy vector: cryo-electron microscopy at $4.5 \AA$ resolution. Structure 2012; 20(8): 1310 20.

http://dx.doi.org/10.1016/j.str.2012.05.004 PMID: 22727812

[12] Subramanian S, Maurer AC, Bator CM, et al. Filling AdenoAssociated Virus Capsids: Estimating Success by CryoElectron Microscopy. Hum Gene Ther 2019; 30(12): 1449-60. http://dx.doi.org/10.1089/hum.2019.041 PMID: 31530236

[13] Lock M, Alvira MR, Wilson JM. Analysis of particle content of recombinant adeno-associated virus serotype 8 vectors by ion-exchange chromatography. Hum Gene Ther Methods 2012; 23(1): 56-64.

http://dx.doi.org/10.1089/hgtb.2011.217 PMID: 22428980

[14] Wang C, Mulagapati SHR, Chen Z, et al. Developing an Anion Exchange Chromatography Assay for Determining
Empty and Full Capsid Contents in AAV6.2. Mol Ther Methods Clin Dev 2019; 15: 257-63.

http://dx.doi.org/10.1016/j.omtm.2019.09.006 PMID: 31720304

[15] Pierson EE, Keifer DZ, Asokan A, Jarrold MF. Resolving Adeno-Associated Viral Particle Diversity With Charge Detection Mass Spectrometry. Anal Chem 2016; 88(13): 6718-25.

http://dx.doi.org/10.1021/acs.analchem.6b00883 PMID: 27310298

[16] Righetti PG, Sebastiano R, Citterio A. Capillary electrophoresis and isoelectric focusing in peptide and protein analysis. Proteomics 2013; 13(2): 325-40. http://dx.doi.org/10.1002/pmic.201200378 PMID: 23180512

[17] Venkatakrishnan B, Yarbrough J, Domsic J, et al. Structure and dynamics of adeno-associated virus serotype 1 VP1unique $\mathrm{N}$-terminal domain and its role in capsid trafficking. $J$ Virol 2013; 87(9): 4974-84.

http://dx.doi.org/10.1128/JVI.02524-12 PMID: 23427155

[18] Goricar B, Peljhan S, Gagnon P. Estimation of empty and full AAV10 particle ratio using the multi-angle light scattering detector. 9th Spring Meeting. March 4-6, 2019; Norfolk, Virginia USA. 2019. 